\title{
FREQÜÊNCIA DE SINTOMAS ASSOCIADOS À ASMA E DOENÇAS ALÉRGICAS EM ADULTOS JOVENS, NA CIDADE DE SANTO ANDRÉ, SP
}

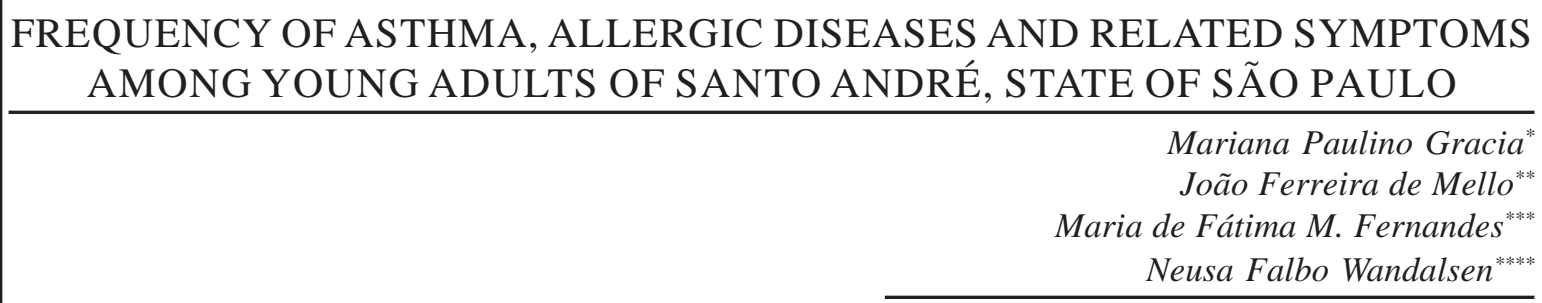

Gracia MP, Mello JFde, Fernandes MdeFM, Wandalsen NF. Freqüência de Sintomas Associados à Asma e Doenças Alérgicas em Adultos Jovens, na Cidade de Santo André, SP. Rev Bras Crescimento Desenvol Hum. 2008; 18(2): 201-208.

Resumo: Objetivo: Determinar a freqüência e a gravidade de sintomas relacionados à asma, à rinite e ao eczema atópico em adultos jovens, empregando o questionário escrito $(\mathrm{QE})$ padronizado e auto-aplicável do ISAAC. Método: 747 estudantes da Faculdade de Medicina do ABC, (Medicina, Enfermagem e Farmácia) foram aleatoriamente escolhidos para responder o QE padrão ISAAC, composto pelos 3 módulos (asma, rinite, eczema). Destes, 600 o fizeram de forma correta e completa. Foram calculadas as freqüências relativas e absolutas das respostas afirmativas às questões analisadas. Resultados: A média de idade da população estudada foi de 26 anos (17- 28), sendo que $67 \%$ eram mulheres. A freqüência de asma ativa (sibilos nos últimos 12 meses) foi 15,3\% e a de asma grave (dificuldade de fala durante as crises) foi $2 \%$. A freqüência de rinite foi $62,1 \%$ ( $35,8 \%$ com sintomas graves), a de rinoconjuntivite foi $33,8 \%$ e a de eczema flexural, $6,6 \%$. Com exceção da asma e seus sintomas, observou-se predomínio no sexo feminino. Os dados analisados indicam a necessidade de estudos adicionais em adultos jovens e a utilização de marcadores para as doenças alérgicas e atópicas.

Palavras-chave: Asma; rinite; eczema; freqüência; ISAAC; alergia.

\section{INTRODUÇÃO}

Acometendo indivíduos de todas as idades, de diferentes níveis sociais e culturais, com graus variados de gravidade e frequiência, a asma é um problema de saúde pública, uma doença inflamatória crônica que se caracteriza por hiperresponsividade das vias aéreas inferiores e limitação variável ao fluxo aéreo ${ }^{1}$. Clinicamente, se manifesta por episódios recorrentes de sibilância, dispnéia, aperto no peito e tosse, particularmente à noite e pela manhã, ao despertar.

A asma, habitualmente, apresenta uma íntima associação com outras doenças, como a rinite alérgica (RA) e o eczema atópico (EA), cujo processo inflamatório também é determinado pela reação alérgica ${ }^{2,3}$. Diversos estudos destacaram a concordância entre as frequiên-

\footnotetext{
Acadêmica de Medicina. Faculdade de Medicina do ABC. Endereço: Al. dos Anapurus, 977 apto.121, Moema - São Paulo-SP CEP 04087-002, E-mail: maripgracia@gmail.com

** Diretor do Serviço de Alergia e Imunologia do Hospital Servidor Público Estadual - São Paulo - SP

*** Chefe do Setor de Ambulatório de Alergia e Imunologia do Hospital Servidor Público Estadual - São Paulo - SP

****** Professora Assistente do Departamento de Pediatria da Faculdade de Medicina do ABC. Coordenadora do Ambulatório de Alergia e Imunologia.

Iniciação Cientifica (PIBIC-Cnpq) no Hospital do Servidor Público Estadual Francisco Morato de Oliveira - FMO. Agência financiadora: Cnpq - Número do processo: 800009/06-1
} 
cias de asma e rinite, dando maior importância aos sintomas nasais na ausência de resfriado ou quando associados a sintomas oculares (rinoconjuntivite alérgica $)^{4,5}$. Com relação ao EA, acomete pacientes com história familiar ou pessoal de atopia, sendo freqüente sua associação com asma e $\mathrm{RA}^{5,6}$.

O EA é mais freqüente em lactentes e em crianças menores, caracterizando-se por eritema, exsudação e vesiculação, principalmente em face e superfícies extensoras dos membros (forma aguda). Também pode acometer adultos e crianças maiores, manifestando-se pela presença característica de escoriações, liquenificação, com predomínio em áreas de flexão ${ }^{7}$.

As manifestações das doenças alérgicas ocorrem em virtude da interação entre fatores genéticos e ambientais. Em indivíduos geneticamente predispostos, a exposição a aeroalérgenos propiciaria a sensibilização, e a exposição crônica levaria à doença. Há relatos que evidenciam que a exposição em níveis elevados de aeroalérgenos apresenta íntima associação com manifestações clínicas de alta gravidade, podendo também favorecer o desencadeamento de crises de asma ou de $\mathrm{RA}^{8}$.

Estudos epidemiológicos, há mais de duas décadas, têm demonstrado um aumento da frequiência de asma e doenças alérgicas em diversos países, causando um grande impacto socioeconômico ${ }^{2}$. O interesse mundial em se estudar a freqüência da asma e os sintomas associados à doença é crescente. Nesse sentido, o questionário do Internacional Study of asthma and allergies in Childhood (ISAAC) foi concebido para estabelecer tal freqüência em crianças com idades entre seis e sete anos e adolescentes entre treze e catorze anos ${ }^{9}$. Não há relatos da aplicação deste instrumento em população adulta em nosso meio, excetuandose o piloto inicial ${ }^{8}$.

Estudos sobre a frequiência de asma e doenças alérgicas na população adulta são poucos e quase nunca relatados. Assim, o objetivo deste estudo é de avaliar a frequiência de sintomas associados à asma e das doenças alérgicas em adultos jovens.

\section{MÉTODO}

\section{Local do Estudo}

Trata-se de estudo transversal, feito por amostragem de conveniência. A população-alvo foi constituída por universitários, com idade entre 17 e 28 anos inclusive, em instituição de ensino em Ciência da Saúde, do município de Santo André, SP.

Santo André localiza-se na Região Metropolitana da Grande São Paulo, integrando um grupo de municípios conhecidos como Região do Grande ABC. Sua superfície abrange uma área de $175 \mathrm{~km}^{2}$ e sua população estimada em 2003 era de 659.294 habitantes, resultando numa densidade demográfica de 3767,39 habitantes $/ \mathrm{km}^{210}$.

\section{International Study of Asthma and Allergies in Childhood (ISAAC)}

A dificuldade em comparar trabalhos realizados em diferentes países, com diferentes instrumentos e métodos de colheita de dados, levou à criação do International Study of Asthma and Allergies in Childhood (ISAAC), projeto desenvolvido com o objetivo de maximizar o valor das pesquisas epidemiológicas em asma e doenças alérgicas em todo mundo ${ }^{11}$. O projeto ISAAC é o mais extensivo estudo internacional sobre asma e doenças alérgicas já realizado. Um dos principais objetivos desse estudo é melhorar a sensibilidade dos questionários sobre sintomas referentes à asma. O questionário é composto por três módulos, contendo 8 questões referentes a asma, 6 questões referentes a rinite e 6 questões referentes ao eczema. A metodologia ISAAC é composta por três fases. Na fase 1 , foram avaliadas as frequiências dos sintomas de asma, rinite e eczema. $\mathrm{Na}$ fase 2, o objetivo principal foi determinar os possíveis fatores etiológicos, empregando metodologias associadas para complementar o diagnóstico, como a aplicação de testes cutâneos e a medida da hiperresponsividade brônquica. A fase 3 compreendeu a repetição da fase 1 , com a finalidade de detectar variações temporais e tendências na frequiência das doenças estudadas.

$\mathrm{O}$ questionário escrito $(\mathrm{QE})$ ISAAC é 
auto-aplicável, de fácil intelecção e capaz de identificar pacientes portadores de asma, rinite alérgica e/ou eczema atópico ${ }^{12}$. Além disso, esse questionário apresenta vantagens sobre outros métodos diagnósticos, como padronização, ausência da necessidade de equipamento especial, independência de variáveis climáticas, baixo custo e boa aceitação ${ }^{13}$.

\section{Descrição do Estudo}

Para o estudo da frequiência de asma e doenças alérgicas foi aplicado o questionário escrito ISAAC fase 1 , pois esta é a fase que se refere à frequiência.

Foram distribuídos 747 questionários aos universitários presentes em cada dia da aplicação, no período de outubro de 2006 a março de 2007, sendo 602 para estudantes da Faculdade de Medicina do ABC do curso de Medicina, do primeiro ao sexto ano; 92, para estudantes do curso de Enfermagem, do primeiro ao terceiro ano e 53 para alunos do curso de Farmácia, do primeiro ao segundo ano.

O questionário escrito ISAAC ${ }^{11}$ foi aplicado na íntegra e respondido pelos próprios estudantes em sala de aula, na presença dos pesquisadores. Não houve recusa para responder ao questionário proposto. Foram excluídos 147
QEs porque estavam com preenchimento incompleto, ilegíveis ou inconsistentes, sendo estudados, portanto, 600 alunos.

Dos quesitos selecionados sobre asma, foram considerados os referentes a sintomas, gravidade e diagnóstico médico da asma: sibilos nos últimos 12 meses (asma ativa); sibilos intensos capazes de limitar-a fala nos últimos 12 meses (asma grave) e asma alguma vez na vida (asma diagnosticada) $)^{4,9}$.

Das questões referentes à rinite, foram consideradas as relacionadas a sintomas nasais, rinoconjuntivite alérgica e formas graves de RA: espirros, coriza e obstrução nasal alguma vez nos últimos 12 meses (rinite); problemas nasais associados aos olhos com prurido e lacrimejamento nos últimos 12 meses (rinoconjutivite alérgica); problema nasal interferindo com a atividade diária (rinite grave) $)^{4,9}$.

Com relação ao EA, foram empregadas as questões referentes a sintomas e gravidade: erupção cutânea que aparece e desaparece por pelo menos 6 meses (eczema); erupção cutânea em locais característicos, como dobras dos cotovelos ou atrás dos joelhos (eczema flexural); rash cutâneo e pruriginoso que interfere com o sono nos últimos 12 meses (eczema grave) ) $^{9,14}$.

O questionário escrito ISAAC fase 1 é apresentado a seguir, em módulos:

\section{MÓDULO 1- ASMA}




\section{MÓDULO 2 - RINITE}

R1 "Alguma vez na vida você teve problemas com espirros ou coriza (corrimento nasal ou obstrução nasal), quando não estava resfriado ou gripado?”

Pode identificar pacientes que não sabem que tem rinite

R2 "Nos últimos 12 meses você teve algum problema com espirros, coriza (corrimento nasal ou obstrução nasal), quando não estava resfriado ou gripado?”

Avalia a possibilidade de crises de rinite no último ano.

R3 "Nos últimos doze meses esse problema nasal foi acompanhado de lacrimejamento_-ou_-coceira nos olhos?" Avalia a possibilidade de uma rinoconjutivite.

R4 "Em qual dos últimos 12 meses esse problema nasal ocorreu?"

Tenta quantificar o número de crises de rinite ao ano e identificar -a sazonalidade.

R5 "Nos últimos 12 meses, quantas vezes suas atividades diárias foram atrapalhadas por esse problema nasal?" Procura identificar a gravidade da rinite.

R6 "Alguma vez na vida você teve rinite?"

Procura identificar a rinite diagnosticada por um médico.

\section{MÓDULO 3 - ECZEMA}

\section{E1 "Alguma vez na vida você teve manchas com coceira na pele (eczema), que apareciam e desapareciam por} pelo menos 6 meses?"

Pode identificar pacientes que não sabem que tem eczema.

E2 "Nos últimos 12 meses você teve essas manchas na pele (eczema)?"

Avalia o número de episódios em um ano.

E3 "Alguma vez essas manchas com coceira afetaram: dobras de cotovelos, atrás de joelhos, na frente de nádegas ou em volta de pescoço, orelhas ou olhos?"

Procura identificar a freqüência de eczema flexural.

E4 “Alguma vez essas manchas com coceira desapareceram completamente nos últimos 12 meses?”

Procura identificar o tempo de evolução da doença.

E5 "Nos últimos 12 meses, quantas vezes, aproximadamente, você ficou acordado a noite por causa dessa coceira na pele?”

Procura identificar a gravidade do eczema.

E6 "Alguma vez você teve eczema?"

Procura identificar o eczema diagnosticado por um médico.

Os dados obtidos foram transcritos para uma planilha de Excel. Foram calculadas as frequiências absolutas e relativas das respostas afirmativas (em porcentagem) aos tópicos analisados. O estudo foi aprovado pelo Comitê de Ética em Pesquisa do Hospital do Servidor Público Estadual sob o $n^{\circ} 009 / 07$. Todos os participantes assinaram o Termo de Consentimento Livre e Esclarecido.

\section{RESULTADOS}

Foram devolvidos 600 questionários preenchidos adequadamente e, destes, 515 (85,8\%) foram respondidos pelos alunos do curso de graduação em Medicina, 61(71,7\%) pelos alunos de Enfermagem e 24 (28,2\%) pelos alunos de Farmácia. A média de idade dos alunos foi de 26 anos. 
Tabela 1-Distribuição dos entrevistados segundo sexo e escolaridade materna.

\begin{tabular}{llll}
\hline \multicolumn{1}{c}{ Característica } & & Número & Porcentagem \\
\hline Sexo & Masculino & 198 & $33,0 \%$ \\
& Feminino & 402 & $67,0 \%$ \\
& Total & 600 & $100,0 \%$ \\
Escolaridade da Mãe & Primeiro Grau & 28 & $4,6 \%$ \\
& Segundo Grau & 95 & $15,8 \%$ \\
& Superior & 477 & $79,5 \%$ \\
& Total & 600 & $100,0 \%$ \\
\hline
\end{tabular}

Tabela 2 - Frequiência (\%) de asma e seus sintomas de acordo com o sexo

\begin{tabular}{llll}
\hline Quest ões & Masculino(n=198) & Feminino(n=402) & Total(n=600) \\
\hline Sibilos alguma vez na vida & 44,4 & 37,8 & 40,0 \\
Sibilos nos últimos 12 meses (asma ativa) & 17,7 & 14,4 & 15,3 \\
Sono alterado por crises de sibilos & 6,0 & 8,0 & 8,0 \\
Fala alterada por crise de sibilos (asma grave) & 3,0 & 1,0 & 2,0 \\
Asma alguma vez na vida (asma diagnosticada) & 13,1 & 12,1 & 12,5 \\
Sibilos aos exercícios físicos & 18,1 & 9,9 & 12,6 \\
Tosse seca noturna sem infecção & 37,3 & 44,7 & 42,3 \\
\hline
\end{tabular}

Tabela 3 - Freqüência (\%) de rinite e seus sintomas de acordo com o sexo

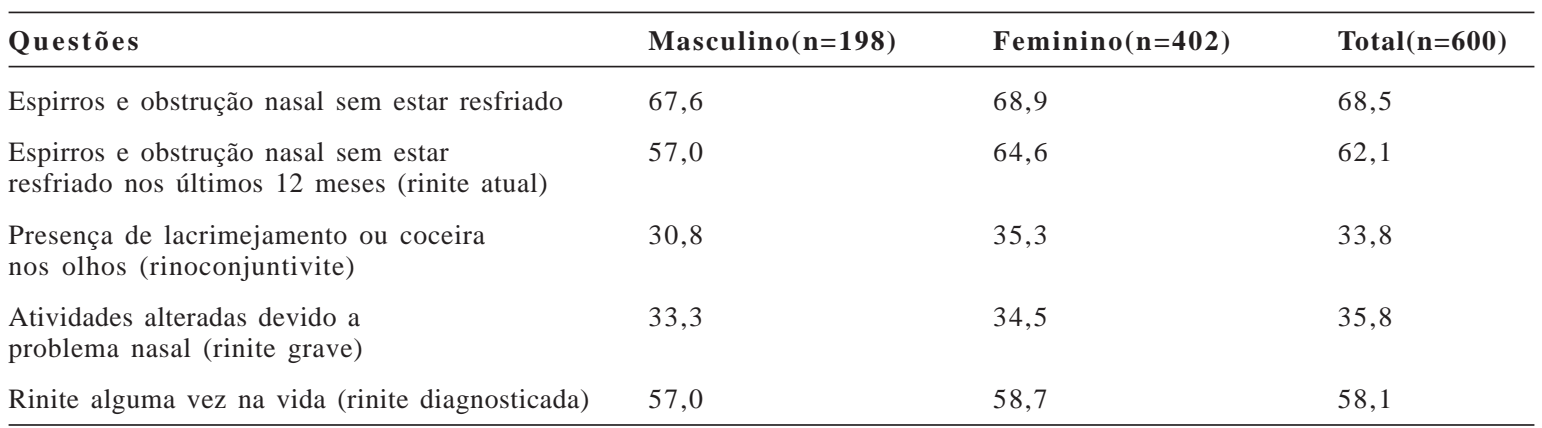

Tabela 4 - Freqüência (\%) de eczema e seus sintomas de acordo com o sexo

\begin{tabular}{llll}
\hline Questões & Masculino(n=198) & Feminino(n=402) & Total $(\mathbf{n}=\mathbf{6 0 0})$ \\
\hline $\begin{array}{l}\text { Manchas com coceira nos últimos } \\
\text { meses (eczema) }\end{array}$ & 15,5 & 16,9 & 16,3 \\
Manchas com coceira nos últimos 12 meses & 9,0 & 12,4 & 11,3 \\
Localização em dobras (eczema flexural) & 5,0 & 7,4 & 6,6 \\
Desaparecimento nos últimos 12 meses & 8,5 & 10,4 & 9,8 \\
Sono alterado por causa da coceira (eczema grave) & 1,5 & 2,4 & 2,1 \\
Eczema alguma vez na vida & 20,7 & 27,8 & 25,5 \\
\hline
\end{tabular}




\section{DISCUSSÃO}

Os estudos de prevalência e freqüência das doenças alérgicas representam um papel fundamental na proposição de medidas para sua prevenção $0^{15,16}$. O estudo ISAAC se constituiu num importante marco da epidemiologia das doenças alérgicas, ficando comprovada a credibilidade e a qualidade do QE ISAAC para a realização de inquéritos em nível mundial ${ }^{17}$. Em nosso trabalho, as tabelas 2 a 4 demonstram que as questões do QE ISAAC representaram instrumento adequado, em termos de aplicabilidade e utilidade, permitindo estabelecer as freqüências de asma ativa $(14,4 \%$ no sexo feminino e $17,7 \%$ no sexo masculino), rinite atual $(64,6 \%$ no sexo feminino e $57,0 \%$ no sexo masculino) e eczema $(12,4 \%$ no sexo feminino e $9 \%$ no sexo masculino).

Numerosos estudos epidemiológicos são relatados em crianças e adolescentes, entretanto a população adulta, em especial a jovem, tal qual descrita neste estudo, tem sido pouco avaliada, dificultando a realização de comparações. Outro fator agravante, é a metodologia empregada nestes poucos trabalhos, desde que o método ISAAC foi pouco utilizado.

O mais completo estudo para asma na população adulta foi o ECRHS (European Community Respiratory Health Survey), que avaliou a frequiência de asma e fatores de risco em 48 centros em 22 países da Europa, Índia e EUA $^{18}$. As questões para determinar a freqüência de asma, rinite e eczema, utilizadas pelo ECRHS, são semelhantes às do QE ISAAC, apresentando os mesmos propósitos ${ }^{18,19}$. Em análise recente, foi demonstrada uma boa correlação entre os resultados do ISAAC (fase I) e do ECRHS (do mesmo país e do mesmo centro), indicando que ambos os questionários possuem valor epidemiológico equivalente para a determinação da freqüência de doenças alérgicas, principalmente da asma ${ }^{20}$.

Na população aqui estudada, constituída por $67 \%$ de indivíduos do sexo feminino, podese observar claramente que a asma e seus sintomas ocorreram com maior frequiência no sexo masculino. A freqüência de sibilos alguma vez na vida, sibilos nos últimos 12 meses (asma ati- va), fala alterada por crise de sibilos (asma grave), asma alguma vez na vida (asma diagnosticada) e sibilos aos exercícios físicos, foi maior no sexo masculino (tabela 2). A amostra, descrita nas tabelas 1 e 2 é semelhante à referida por Andrade et $\mathrm{al}^{8}$, que analisaram igualmente população de estudantes universitários, na cidade de São Paulo, destacando-se as diferenças entre os sexos, quanto aos sintomas de asma.

Vichyanond et $\mathrm{al}^{21}$; empregaram o QE ISAAC em universitários em Bangkok, observando uma frequiência de sibilos nos últimos doze meses de $10,1 \%$ e de asma alguma vez de $8,8 \%$. Em nosso estudo, os resultados referentes a tais questões foram, respectivamente, $15,3 \%$ e $12,5 \%$. Com relação ao sexo, nos universitários de Bangkok ocorreu predomínio de asma ativa e asma diagnosticada no sexo masculino, da mesma forma que na população por nós estudada. Por outro lado, no estudo ECRHS ${ }^{18}$, a frequiência de sibilos nos últimos 12 meses foi de 4-32\%, e de asma alguma vez na vida foi de $2-12 \%$.

Em nosso estudo, ao se analisar variáveis, como espirros e obstrução nasal sem estar resfriado, nos últimos 12 meses (rinite atual), presença de lacrimejamento ou coceira nos olhos (rinoconjutivite), atividades alteradas devido a problema nasal (rinite grave) e rinite alguma vez na vida (rinite diagnosticada), observou-se predomínio do sexo feminino (tabela 3), o mesmo ocorrendo com manchas com coceira nos últimos 12 meses (eczema), manchas com coceira nos últimos 12 meses com localização em dobras (eczema flexural), desaparecimento das manchas nos últimos 12 meses, sono alterado por causa da coceira (eczema grave) e eczema alguma vez na vida (tabela 4).

De acordo com os vários centros que aplicaram o protocolo ISAAC, pode-se concluir que a freqüência de RA vem aumentando em diversos países ${ }^{15}$. Na presente análise, a frequiência de rinite atual foi elevada $(62,1 \%)$ semelhante à encontrada por Vichyanond et $\mathrm{al}^{21}(61,9 \%)$.

É importante salientar que, ao se avaliar estudantes universitários, que apresentem melhores condições socioeconômicas, a frequiência de RA é maior, em comparação a populações menos favorecidas, de acordo com estudos rea- 
lizados na África do $\mathrm{Sul}^{22}$ e no Brasil ${ }^{23}$.

O predomínio da RA no sexo feminino, bem como maior freqüência no inverno, foram observados em nosso estudo, assim como em diversas pesquisas $^{24}$. Leynaert et $\mathrm{al}^{25}$ comprovaram que a relação entre a gravidade da RA e piora da qualidade de vida é mais evidente em mulheres, conforme os dados mostrados na tabela 3 .

Em nossa amostra, a frequiência de EA $(6,6 \%)$ foi semelhante à encontrada por Vichyanond et $\mathrm{al}^{21}(9,4 \%)$, que também utilizaram o "critério combinado" para diagnóstico de EA, conforme proposto pelo ISAAC ${ }^{26}$.
De acordo com resultados deste estudo, concluímos que a doença alérgica mais freqüente em adultos jovens é a rinite, seguida por asma ativa, ou seja, sibilos nos últimos 12 meses, e eczema flexural. Com relação à gravidade, a doença mais freqüente foi a rinite, sendo que asma e o eczema graves apresentaram freqüências semelhantes.

Estes dados nos fazem acreditar na necessidade da realização de estudos adicionais em adultos jovens, em nosso meio, utilizando outras ferramentas importantes para identificar portadores de doenças alérgicas e atopia.

\begin{abstract}
Aim: To determine the Frequence and severity of symptoms related to asthma, rhinitis and atopic eczema in young adults, using the standard self-applicable ISAAC written questionnaire (WQ). Method: 747 students of Faculdade de Medicina do ABC (Medicine, Nursing and Pharmacy) were randomly chosen to answer the WQ. Six hundred students correctly and completely answered the WQ. Relative and absolute frequencies of affirmative answers to the analyzed questions were calculated. Results: The mean age of the studied population was 26 years (17-28) and 67\% were women. The prevalence of active asthma (wheezing in the last 12 months) was $15.3 \%$ and of severe asthma (speech difficulty during the crisis), $2 \%$. The Frequence of rhinitis was $62.1 \%$ (35.8\% with severe symptoms), of rhinoconjunctivitis, $33.8 \%$, and of flexural eczema, $6.6 \%$. With the exception of asthma and its symptoms, there was predominance of these diseases in females. The analyzed data indicate the need for further studies in young adults and the use of markers for allergic and atopic diseases.
\end{abstract}

Keywords: Asthma; rhinitis; eczema; prevalence; ISAAC; allergy.

\section{REFERÊNCIAS}

1. Ferrari PF, Rosário Filho NA, Ribas LFO, Callefe LG. Frequiência de asma em escolares de Curitiba-projeto ISAAC (Internacional Study of Asthma and Allergies in Childhood).J. Pediatr(Rio J.). 1998; 74(4); 299-305.

2. Ellwood P, Asher MI, Beaslley R, Clayton TO, Stewart AW. ISAAC Steeriing Committee. The Internacional Study of Asthma and Allergies in Childhood (ISAAC): phase three rationale and methods. Int J Tuberc Lung Dis 2005 Jan;9(1):10-6.

3. Gerritsen J, Koëter GH, Monchy JGR, Knol K. Allergy in subjects with asthma from childhood to adulthood. J allergy Clin Immunol 1990; 85:116-125.

4. Worldwide variations in the prevalence of asthma symptoms: The International Study of Asthma and Allergies in Childhood (ISSAC). Eur Respir J. 1998; 12:315-35.

5. Strachan D, Sibbald B, Weiland S, ait-Khaled N, Anabwani G, Anderson R, et al. Worldwide variations in the prevalence of symptoms of allergic rhinoconjunctivitis in children: The International Study of Asthma and Allergies in Childhood (ISSAC). Pediatr Allergy immunol. 1997;8:161-76.

6. Camelo-Nunes IN, Wandalsen GF, Melo KC, Naspitz CH, Solé D. Freqüência de eczema atópico e sintomas relacionados entre estudantes. J.Pediatr. (Rio J.) 2004; 80(1):60-4.

7. Castro APM, Solé D, Rosário Filho NA, Jacob CMA, Rizzo MCFV, Fernandes MFM, Vale SOR. Guia Prático para o Manejo da Dermatite Atópica-opinião conjunta de especialistas em alergologia da Associação Brasileira de Alergia e Imunopatologia e da Sociedade Brasileira de Pediatria. Rev. bras. alerg. imunopatol. 2006;29(6):268-282.

8 Andrade SRG, Rodrigues CE, Castro MA, Nunes ICC, Solé D, Kalil J, Castro FFM.

Freqüência e fatores de risco para asma em estudantes de Veterinária e Medicina. Rev. Bras. Alerg. Imunopatol. 2005; 28(2); 89-93. 
9. Asher MI, Keil U, Anderson HR, Beasley R, Crane J, Martinez F, et.al. Intenational Study of Asthma and Allergies in Childhood (ISAAC): rationale and methods. Eur Respir. J. 1995;8:48391.

10. Prefeitura municipal de Santo André - Governo eletrônico. Disponível em URL: http:// www.santoandre.sp.gov.br [ 2007 mar 15]

11. Asher MI, Weiland SK. The Internacional Study of Asthma and Allergies in Childhood (ISAAC). ISAAC Steeriing Committee. Clin Exp. Allergy. 1998 Nov; 28 Suppl 5:52-66; discussion 90-1.

12. Solé D, Wandalsen GF, Camelo-Nunes IN, Naspitz CH, ISAAC-Grupo Brasileiro.

Freqüência de sintomas de asma, rinite e eczema atópico entre crianças e adolescentes brasileiros identificados pelo Internacional Study of Asthma and Allergies in Childhood (ISAAC) - Fase 3. J.Pediatr. (Rio J.) vol. 82 no.5 Porto Alegre Sept./Oct.2006.

13 Maia JGS, Marcopito LF, Amaral AN, Tavares BF, Lima e Santos FAN. Freqüência de asma e sintomas asmáticos em escolares de 13 e 14 anos de idade. Rev. Saúde Pública 2004; 38(2): 292-9.

14. Willams H, Robertson C, Steawart A, AitKhaled N, Anabwani G, Anderson R, et. al. Worldwide variations in the prevalence of symptoms of atopic eczema in the International Study of Asthma and Allergies in Childhood (ISAAC). J Allergy Clin Immunol.1999;103:12538.

15. ISAAC Steering Committee (The International Study of Asthma and Allergies in Children Steering Committee). Worldwide variation in prevalence of Symptoms of asthma, allergic rhinoconjunctivitis, and atopic eczema: ISAAC. Lancet 1998;351:1225-32

16. Solé D, Yamada E, Vanna AT, Costa-Carvalho BT, Naspitz CK. Prevalence of asthma and related symptoms in school-age children in São Paulo, Brazil International Study of Asthma and Allergies (ISAAC). J Asthma 1999;36:205-12.

17. Solé D, Vanna AT, Yamada E, Rizzo MCV, Naspitz CK. International Study on Asthma and Allergies in Children (ISAAC) written questionnaire: validation of the asthma component among Brazilian children. Invest Allergol Clin Immunol 1998;8:376-82.

18. Burney P,Chinn S, JarvisD,Luczynska C,Lai E.Variations in the prevalence of respiratory symptoms, self-reported asthma attacks and use of asthma medication in the EuropeanCommunity Respiratory Health Survey (ECRHS).Eur Respir J1996; 9: 687^ 695.

19. Beasley R, Keil U, von Mutius EV, Pearce N.Worldwide variation in prevalence of symptoms of asthma, allergic rhinoconjunctivitis, and atopic eczema. ISAAC.Lancet $1998 ; 351: 1225^{\wedge} 1232$.

20. Pearce N, Sunyer J,Cheng S, etal.Comparison of asthma prevalence in the ISAAC and the ECRHS.Eur Respir J 2000; 16: 420^ 426.

21. Vichyanond P, Sunthornchart S, Singhirannsorn, Ruangrat S, Kaewsombo ON, Visitsunthorn N. Prevalence of asthma, allergic rhinitis and eczema among university students in Bangkok. Respir Med, 2002;96:34-38.

22. Mercer MJ, Joubert G, Ehrlich RI, Nelson H, Poyser MA, Puterman A, et al. Socioeconomic status and prevalence of allergic rhinitis and atopic eczema symptoms in young adolescents. Pediatr Allergy Immunol. 2004;15:234-41.

23. Nascimento-Carvalho CM, Rocha H, Benguigui Y. Effects of socioeconomic status on presentation with acute lower respiratory tract disease in children in Salvador, Northeast Brazil. Pediatr Pulmonol. 2002;33:244-8.

24. Shamssain MH, Shamsian N. Prevalence and severity of asthma, rhinitis, and atopic eczema in 13 to 14-year-old schoolchildren from the northeast of England. Ann Allergy Asthma Immunol. 2001;86:428-32.

25. Leynaert, Neukirch, Liard, et al. : Quality of Life in Rhinitis and Asthma. Am. J. Respir. Crit. Care Med., Volume 162, Number 4, October 2000, 1391-1396.

26. Sole D, Camelo-Nunes IC, Rosario NA, Freitas LS, Britto M, Melo K, et al. Prevalence of asthma, rhinoconjuntivitis and atopic eczema among Brazilian adolescents. Comparison between ISAAC phases I and III [abstract]. J Allergy Clin Immunol. 2004;113:1030. 